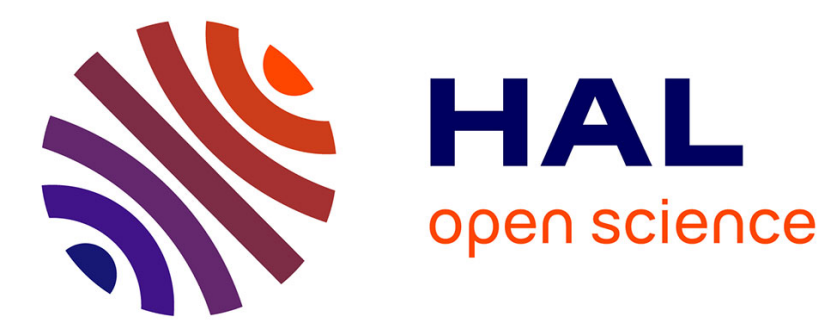

\title{
Predicting droplet velocity in a Hele-Shaw cell
}

Benjamin Reichert, Isabelle Cantat, Marie-Caroline Jullien

\section{To cite this version:}

Benjamin Reichert, Isabelle Cantat, Marie-Caroline Jullien. Predicting droplet velocity in a Hele-Shaw cell. Physical Review Fluids, 2019, 4 (11), 10.1103/PhysRevFluids.4.113602 . hal-02396701

\section{HAL Id: hal-02396701 \\ https://hal.science/hal-02396701}

Submitted on 6 Dec 2019

HAL is a multi-disciplinary open access archive for the deposit and dissemination of scientific research documents, whether they are published or not. The documents may come from teaching and research institutions in France or abroad, or from public or private research centers.
L'archive ouverte pluridisciplinaire HAL, est destinée au dépôt et à la diffusion de documents scientifiques de niveau recherche, publiés ou non, émanant des établissements d'enseignement et de recherche français ou étrangers, des laboratoires publics ou privés. 


\title{
Predicting droplet velocity in a Hele-Shaw cell
}

\author{
Benjamin Reichert ${ }^{1}$, Isabelle Cantat $^{2}$, and Marie-Caroline Jullien ${ }^{1,2}$ \\ ${ }^{1}$ Gulliver UMR CNRS 7083, PSL Research University, \\ ESPCI Paris, 10 rue Vauquelin, F-75005 Paris, France \\ ${ }^{2}$ Univ Rennes, CNRS, IPR (Institut de Physique \\ de Rennes) - UMR 6251, F-35000 Rennes.
}

(Dated: December 6, 2019)

\begin{abstract}
We study the motion of low viscous non-wetting droplet in a Hele-Shaw cell, while it is pushed by an external phase of imposed flow rate, at low capillary numbers. In this regime, the droplet's mobility, defined as the ratio between the droplet velocity and the external phase mean velocity, evolves non linearly with the capillary number, a signature of the different dissipation mechanisms at play. Experiments are performed with surfactant free air bubbles in fluorinated oil, and with surfactant laden fluorinated oil droplets in water. We propose a model based on a power balance which takes into account the dissipation in the thin wetting film trapped between the bubble (or the drop) and the channel wall. The full topography of this thin film is obtained theoretically for the bubble case. By contrast, the presence of surfactants in the drop case induces uncontrolled boundary conditions at the interface, thus imposing to use the experimental topography measured in the previous paper [Reichert et al., J. Fluid. Mech., 850 p.708 (2018)]. Remarkably, the model reproduces the experimental velocities and shows that the velocity can be strongly affected by a stagnant cap effect at the rear of the drop, even if localized in less than a few percents of the total film area.
\end{abstract}




\section{INTRODUCTION}

The important development of droplet-based microfluidics these last ten years has rekindled the interest in the study of droplets and bubbles dynamics in micro-channels [1-5]. Predicting the stationary speed $U_{d}$ of a droplet (or bubble) squeezed between two plates, while it is pushed by an external phase of set velocity $U_{f}$, is a problem which was addressed in the pioneering work of Taylor \& Saffman for bubbles in a pure Newtonian fluid [6]. Assuming the bubble has a cylindrical shape, they found $U_{d}=2 U_{f}$, systematically overestimating experimental data $[7,8]$. Two sources of dissipation have been identified for a non wetting droplet: (i) in the wetting film trapped between the wall and the drop ; and (ii) in the dynamical meniscus, $i$. e. the transition region between the drop side and the wetting film [9]. Models considering dissipation (i), with a uniform interfacial velocity equal to $U_{d}$ underestimate the drop velocity $[8,10]$. In the literature, models accounting for dissipation (ii) with a stress-free condition at the interface (the film is not sheared) obtain reliable velocity predictions, but only for surfactant free systems at low capillary numbers [11]. This last case and its extension to viscous droplets have also been investigated using direct 3D numerical simulations [12], but for droplet velocities compelled to higher values than our experimental

ones. Indeed, the very high aspect ratio between the wetting film thickness and the channel height at small droplet velocity observed experimentally [13] prevents from solving the flow using direct numerical simulation in the entire flow domain $[2,12]$.

In more realistic situations, contaminants can modify the interface rheology and consequently the drop velocity. The induced Marangoni effects, and more specifically the surfactant-induced stiffening of the interface, have been considered in theoretical and numerical models [14-21]. The major difficulty relies in the determination of the relevant microscopic parameters governing the surfactant transport and their interaction with the interface, preventing from building a model able to reproduce all the reported experimental data. However, we recently showed that the local interfacial velocity can be reliably extracted from the wetting film thickness map $(i$. e. the value of the film thickness at each point), even if indirectly, and without the need of a surfactant transport constitutive law [13]. The knowledge of these surface properties (thickness map in the whole thin wetting film and surface velocity field in the same domain) are key elements to derive the droplet velocity. 
TABLE I. Properties of system 1 (top) and system 2 (bottom).

\begin{tabular}{|c|c|c|c|c|c|c|c|}
\hline Phase & Working liquids & $\begin{array}{c}\text { Viscosity } \\
\text { mPas }\end{array}$ & $\begin{array}{c}\text { Surfactant } \\
{[\text { Surfactant }](\mathrm{mM})}\end{array}$ & $\begin{array}{c}\text { Surface tension } \\
\mathrm{mN} \mathrm{m}^{-1}\end{array}$ & $\begin{array}{c}\text { Value of droplet } \\
\text { radius }(\mu \mathrm{m})\end{array}$ & $\begin{array}{c}\text { Range of } C a \\
\left(=\eta_{o} U_{d} / \gamma\right)\end{array}$ & $\begin{array}{l}\text { boundary } \\
\text { condition }\end{array}$ \\
\hline $\begin{array}{l}\text { continuous } \\
\text { dispersed }\end{array}$ & $\begin{array}{l}\text { Fluorinated oil } \\
\text { Air }\end{array}$ & $\begin{array}{c}\eta_{o}=4.5 \\
\eta_{i}=0\end{array}$ & $\begin{array}{c}\text { No surfactant } \\
0\end{array}$ & $\gamma=15$ & $77-100-160$ & $5 \times 10^{-4}-5 \times 10^{-3}$ & stress-free \\
\hline $\begin{array}{l}\text { continuous } \\
\text { dispersed }\end{array}$ & $\begin{array}{l}\text { DI water }+\mathrm{NaCl} \\
\text { Fluorinated oil }\end{array} \mid$ & $\begin{array}{l}\eta_{o}=2.5 \\
\eta_{i}=0.64\end{array}$ & $\begin{array}{c}\text { C10TAB } \\
990\end{array}$ & $\gamma=15$ & $80-100$ & $2.3 \times 10^{-4}-4.8 \times 10^{-3}$ & $\begin{array}{l}\text { stress-free at the front } \\
\text { sliding at the back }\end{array}$ \\
\hline
\end{tabular}

In this paper, we quantitatively relate the drop velocity to the thickness map, using a fine identification of all the relevant dissipative contributions, with the most appropriate approximation level in each fluid region. The model is first validated in the reference situation of an air bubble in pure oil (System 1), for which the thickness map is known theoretically, and governed by a stress-free condition at the interface [11]. Then we consider oil droplets in an aqueous surfactant solution (System 2). In this case the use of the previous, stress-free, thickness map overestimates $U_{d}$ by a factor 1.3. We use instead the experimental thickness map $h^{\exp }$ obtained in [13] and get a quantitative prediction for the drop velocity. This map $h^{\exp }$ exhibits the signature of an interface stiffening close to the rear meniscus, but only in $5 \%$ of the total wetting film area. An important result of the paper is thus the sensitivity of the drop velocity to very localized interface stiffening, making it especially difficult to predict. For this reason, the droplet velocity value can be a strong indication of an interface stiffening, even locally.

\section{EXPERIMENTAL SETUP}

The droplets/bubbles are generated at a T-junction in a polydimethylsiloxane (PDMSSylgard 184) microfluidic system, and enter a $2500 \times 9000 \mu \mathrm{m}$ Hele-Shaw cell of thickness $2 H_{o}=14 \mu \mathrm{m}$. The microfluidic chip is shown in Fig. 1.a. The upper part of the chamber is made of PDMS and is fabricated using soft photo-lithography [22]; the top wall of the chamber is stiffened by including a glass slide within the PDMS matrix in order to ensure that the channel does not deform even at the highest pressures applied [23]. The PDMS part is further permanently bonded to a glass slide via oxygen plasma. The dispersed phase is injected by mean of a pressure controller (Fluigent). The flow rate of the external phase is imposed using a syringe pump (Nemesys controler) and measured at the outlet of the cavity 
by mean of a flow-well (Fluigent). The external phase is mainly fed by the channel comb, which plays the role of an accelerator allowing to impose the global flow rate in the cavity and to adjust the distance between successive droplets to avoid hydrodynamical interactions. The comb shape prevents the droplets from splitting. As, far from the drop, this flow is uniform in the transverse cell section ( $y$ direction), the depth average velocity of the external phase $U_{f}$ can be deduced from the measured flow rate. The droplet velocities are measured using spatio-temporal plots along their direction of motion. The highest bubble/droplet velocity reachable in our experiment is bounded by the maximum pressure that the pressure controller can deliver (1 bar). Two working liquids are studied: 1) System 1 considers surfactant free air bubbles in fluorinated oil and, 2) System 2 focuses on fluorinated oil droplets in an aqueous solution containing surfactants (same system as in [13]). The working liquids physico-chemical properties and the experimental specifications are summarized in table I. We focus on the steady regime, far from the cavity inlet and outlet. For both systems a thin film of continuous phase separates the bubble/drop from the wall.
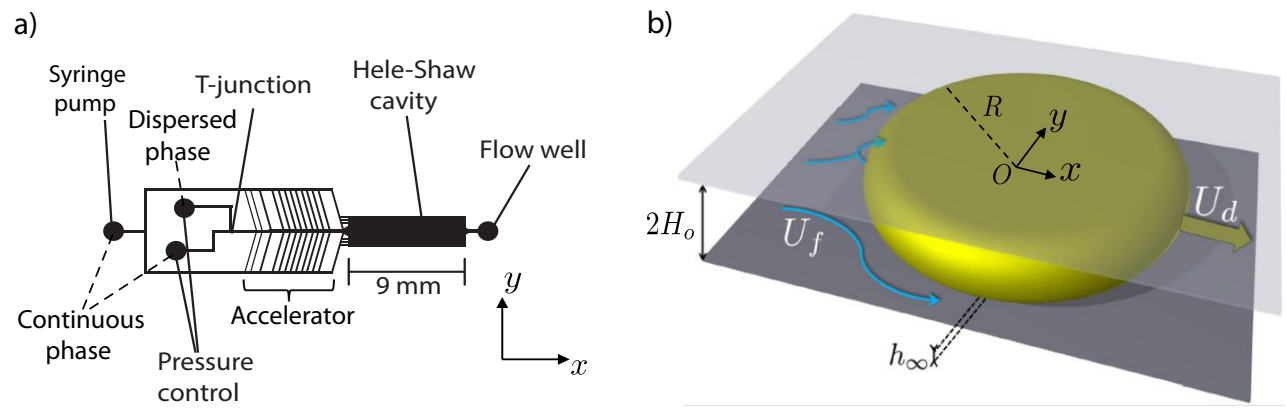

FIG. 1. (a) Design of the microfluidic chip: droplets/bubbles are generated at the T-junction and flow towards the Hele-Shaw cavity. In order to avoid hydrodynamical interactions between successive droplets, external phase is added through the comb shape channels located aside of the main channel. (b) Pancake-shaped droplet/bubble moving in a Hele-Shaw cell: $2 H_{o}$ is the cell thickness, $U_{f}$ and $U_{d}$ are respectively the external fluid and the droplet/bubble velocity, $h_{\infty}$ is the lubrication film thickness. 


\section{EXPERIMENTAL RESULTS}

Fig. 2 displays the mobility $U_{d} / U_{f}$ as a function of the capillary number $C a=\eta_{o} U_{d} / \gamma$ for System 1 and System 2. The value of an experimental point is an average performed on ten bubble/droplet velocities of same radius. The experimental error mainly stems from the measurement of the flow rate by the flow well (Fluigent) at the exit of the channel.

The radius of the bubble/droplet is determined by the geometry of the T-junction [24]. In the Hele-Shaw cell, the droplet adopts a pancake like shape (see Fig. 1.b). It refers to a droplet which has a radius $R$ verifying $\varepsilon=H_{o} / R \ll 1$, experimentally $\varepsilon \leq 1 / 10$. Since the relative variation of the droplet/bubble contour radius is of the order of $\delta R / R \sim 3 \%$, these objects are considered as circular in this study.

Fig. 2.a corresponds to the data obtained for air bubbles in fluorinated oil (System 1) and the prediction reported by Taylor \& Saffman $\left(U_{d} / U_{f}\right)_{\mathrm{TS}}=2$ for circular pancake bubble is sketched as a solid line [6]. The data obtained for oil droplets in water containing surfactants (System 2) are displayed on Fig. 2.b. Gallaire et al. extended the Taylor \& Saffman mobility to viscous droplets and obtained $\left(U_{d} / U_{f}\right)_{\mathrm{TSV}}=2 /\left(1+\eta_{i} / \eta_{o}\right)$, represented as a solid line on Fig. 2.b [25]. As a general comment for both sets of data, the mobility increases with the capillary number and with the radius of the droplet/bubble. More interestingly, the previous model, based on the viscous dissipation due to the Poiseuille flow inside and outside the bubble/drop only, overestimates the experimental mobility for both systems. Additional sources of dissipation stem from the confined region separating the droplet interface from the channel wall $[9,11]$, which are taken into account in the model developed below.

\section{DROPLET/BUBBLE VELOCITY MODELING}

In the following, we propose a model based on a power balance considering the different sources of dissipation [26]. In the bubble case, the boundary conditions at the interface are known, and the dissipation can be determined by solving the Stokes equations with the appropriate approximations. In contrast, for the drop case, we showed in a previous study that the presence of surfactant at the interface induces Marangoni effects which can not be determined ab initio without a better knowledge of the their transport properties. The boundary condition can however be deduced from the measurement of the thickness profile 
a)

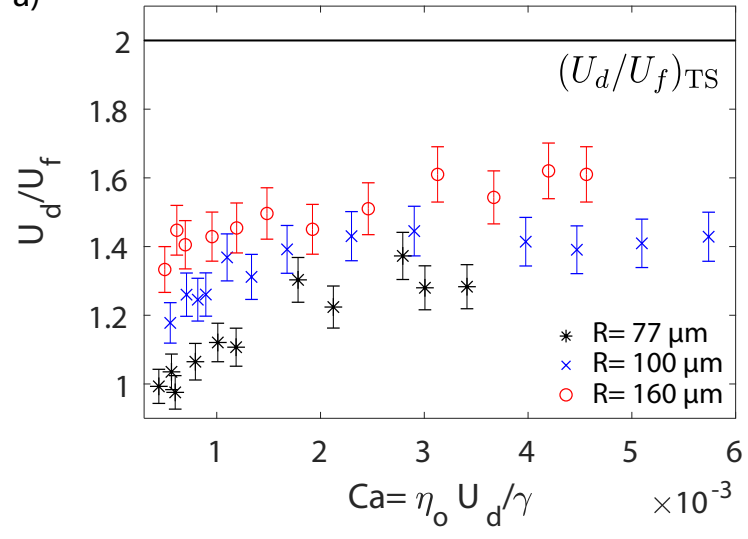

b)

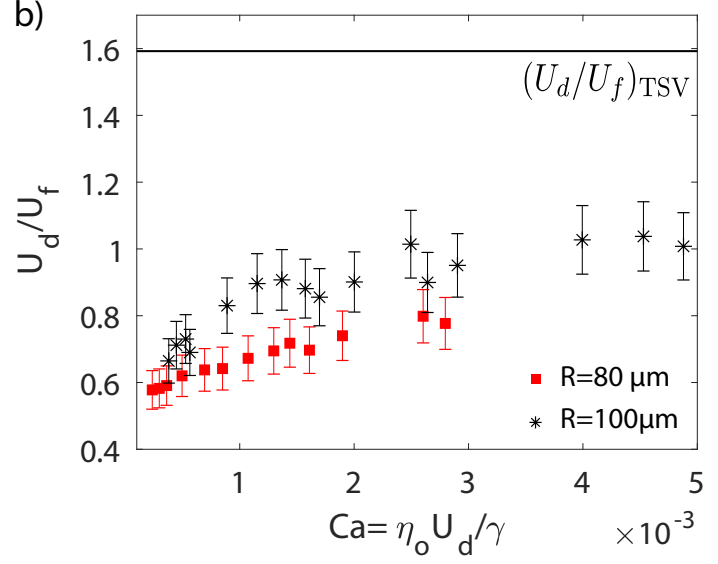

FIG. 2. Mobility $U_{d} / U_{f}$ as a function of $C a$ for different radii. (a) bubbles (System 1) with $R=160 \mu \mathrm{m}(\circ), R=100 \mu \mathrm{m}(\times)$ and $R=77 \mu \mathrm{m}(\star)$. The horizontal line is Taylor \& Saffman prediction $\left(U_{d} / U_{f}\right)_{\mathrm{TS}}=2[6]$. (b) droplets (System 2) with $R=100 \mu \mathrm{m}(\star)$ and $R=80 \mu \mathrm{m}(\mathbf{\bullet})$. The horizontal line is Gallaire's prediction $\left(U_{d} / U_{f}\right)_{\mathrm{TSV}}=2 /\left(1+\frac{\eta_{i}}{\eta_{o}}\right)$ with $\eta_{i} / \eta_{o}=0.64 / 2.5=$ $0.26[25]$.

[13]: using these experimentally obtained boundary conditions as an input in our dissipation model, we are able to predict accurately the drop velocity.

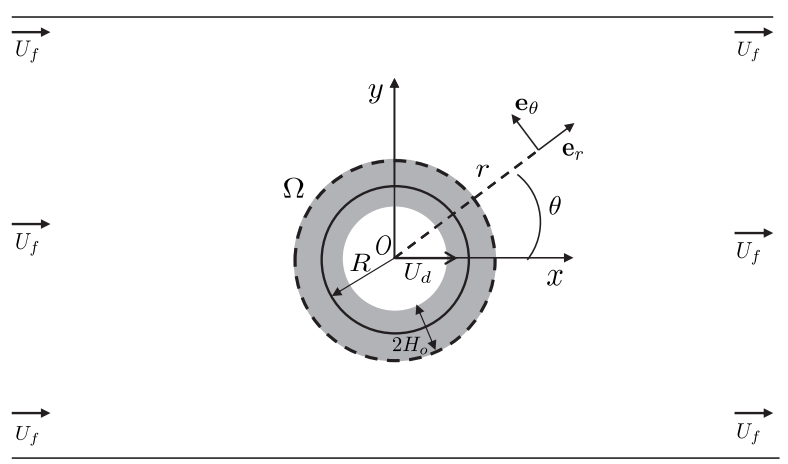

FIG. 3. Cross-section of the bubble/droplet for $z=H_{o}$. The energy balance is performed within the cylindrical control volume $\Omega$ of radius $\left(R+H_{o}\right)$ and height $2 H_{o}$.

In both cases, the dissipation is determined in a cylindrical control volume $\Omega$ of radius $R_{\Omega}=R+H_{o}$ and height $2 H_{o}$ surrounding the droplet, see Fig. 3 and 4 . Different regions are described at different levels of approximation: (i) far from the drop boundary the flow is known analytically (inside and outside the drop) ; (ii) in the static meniscus (close to the 
drop boundary, far from the wall) scaling laws show that dissipation is negligible; finally the lubrication equations are solved numerically (iii) in the dynamical meniscus and (iv) in the thin film. The droplet motion is studied in the laboratory frame of reference, using the ortho-normal spatial system $\left(O, \mathbf{e}_{x}, \mathbf{e}_{y}, \mathbf{e}_{z}\right)$ attached to the drop as well as the cylindrical coordinates $(r, \theta, z)$, with $(O x)$ as the polar axis, see Fig. 3 and 4 . The whole study is performed at first order in $\varepsilon=H_{o} / R$.

\section{A. Flow around a moving cylinder and control volume}

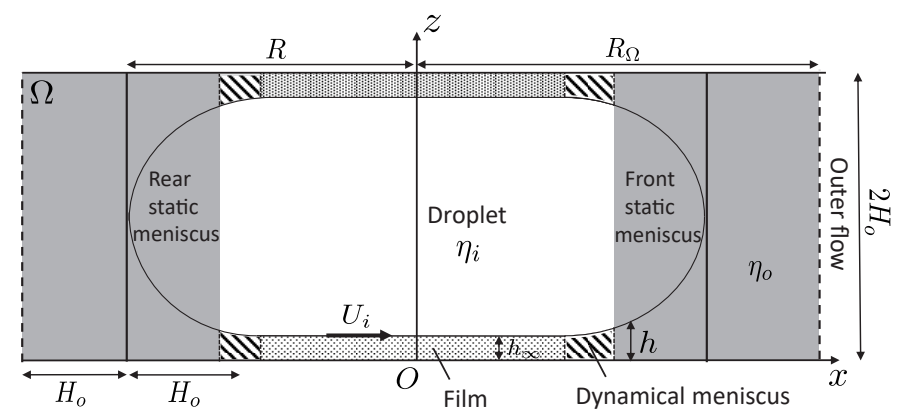

FIG. 4. Transverse cross-section of the droplet for $y=0$, with the different domains and notations used in the text. The central part of the film and the dynamical meniscus constitute the lubrication film, in which most of the dissipation occurs. The size of the dynamical meniscus is exaggerated for the sake of clarity.

The Reynolds number, built as $R e=\rho U_{d} H_{o} / \eta_{o}<10^{-4}$, is small enough to neglect inertia in the whole problem. The flow thus obeys the Stokes equation, and, in the lubrication limit, the velocity profile is

$$
\mathbf{u}(x, y, z)=\frac{3}{2} \mathbf{V}(x, y) \frac{z\left(2 H_{o}-z\right)}{H_{o}^{2}}
$$

with $\mathbf{V}(x, y)$ the velocity averaged over the thickness of the Hele-Shaw cell, verifying

$$
\begin{aligned}
\nabla P+\frac{3 \eta_{o}}{H_{o}^{2}} \mathbf{V} & =0 \\
\nabla \cdot \mathbf{V} & =0 .
\end{aligned}
$$

The flow around a cylinder moving at velocity $U_{d}$ is classically given by the superposition of a uniform flow of velocity $U_{f} \mathbf{e}_{x}$ far from the cylinder, and a dipolar disturbance flow of dipole moment $\mathbf{m}=2 \pi R^{2}\left(U_{d}-U_{f}\right) \mathbf{e}_{x}$ in the vicinity of the cylinder $[12,27]$ : 


$$
\left\{\begin{array}{l}
V_{r}(r, \theta)=U_{f} \cos (\theta)-\left(U_{f}-U_{d}\right) \frac{R^{2}}{r^{2}} \cos (\theta) \\
V_{\theta}(r, \theta)=-U_{f} \sin (\theta)-\left(U_{f}-U_{d}\right) \frac{R^{2}}{r^{2}} \sin (\theta)
\end{array}\right.
$$

The associated pressure field writes:

$$
p_{\text {out }}(r, \theta)=-\frac{3 \eta_{o}}{H_{o}^{2}}\left[r U_{f} \cos (\theta)+\left(U_{f}-U_{d}\right) \frac{R^{2}}{r} \cos (\theta)\right]
$$

The velocity field around a circular pancake drop or bubble is equal to this reference field, far enough from the boundaries. Indeed, the curvature in the plane $\left(O, e_{r}, e_{z}\right)$ and the tangential velocity at the interface induce specific 3D corrections in a ring of lateral extension of the order of the cell thickness $H_{o}$ [28]. For this reason, the control volume $\Omega$ is determined such that eqs. (4) and (5) are valid on its lateral frontier. The domain $\Omega$ is thus a cylinder of radius $R_{\Omega}=R+H_{o}$, centered on the bubble/drop center, bounded by the top and bottom walls.

\section{B. Power balance in the control volume}

As the kinetic energy is negligible in the problem, the energy conservation imposes that the power delivered by the external forces $\mathcal{P}$ at the boundary of $\Omega$ is balanced at each time with the viscous dissipation rate $D_{\Omega}$ in this control volume, i.e.

$$
\mathcal{P}=D_{\Omega}
$$

First, the external power provided to our open system $\Omega$ is determined. Both the top and bottom boundaries of $\Omega$ are immobile walls which do not provide mechanical work. On the contrary, on the lateral boundaries the viscous and pressure forces must be considered. The viscous stress, obtained by the derivation of the velocity field (4), scales as $\eta_{o} U_{f} / R$; and

the pressure, given by eq. (5) scales as $\eta_{o} U_{f} R / H_{0}^{2}$. At leading order in $\varepsilon=H_{0} / R$, only the pressure contribution is considered. The power $\mathcal{P}$ injected in $\Omega$ through the lateral surface $S$ is thus given by (using eqs. (1), (4), (5)) :

$$
\begin{aligned}
\mathcal{P} & \simeq-\int_{S} p_{\text {out }} \mathbf{u} \mathrm{d} \mathbf{S} \\
& \simeq \frac{6 \eta_{o} \pi\left(R+H_{o}\right)^{2}}{H_{o}}\left[U_{f}^{2}-\left(U_{f}-U_{d}\right)^{2}\left(\frac{R}{R+H_{o}}\right)^{4}\right],
\end{aligned}
$$


which can be written, at leading order in $\varepsilon=H_{o} / R$, as

$$
\mathcal{P} \simeq \frac{6 \eta_{o} \pi R^{2}}{H_{o}}\left(2 U_{f}-U_{d}\right) U_{d}
$$

The power balance eq. (6) and the relation (8) finally provide the expression of the bubble/drop mobility as a function of the viscous dissipation in $\Omega$ [26]:

$$
\beta=\frac{U_{d}}{U_{f}}=\frac{2}{1+\frac{D_{\Omega}}{D_{\mathrm{ref}}}} .
$$

with $D_{\text {ref }}=6 \pi \eta_{o} R^{2} U_{d}^{2} / H_{o}$ the viscous dissipation which would occur in $\Omega$ if the whole system were filled with the continuous phase (no interface).

In the case of a bubble, the volume $\Omega$ contains mainly an inviscid gas, and Taylor \& Saffman model disregards the dissipation in the residual liquid phase in $\Omega$, around the bubble. In that case, $D_{\Omega}=0$ and eq. (9) directly leads to $U_{d}=2 U_{f}[6]$.

The delicate part of this study is the determination of $D_{\Omega}$ since it depends on the shape and the boundary condition at the interface of the bubble/droplet. In all our experimental cases, $0.5<U_{d} / U_{f}<1.5$, so $2 U_{f}-U_{d} \sim U_{d} \sim U_{f}$ and the injected power eq. (8) is thus of the order of $\eta_{o} R^{2} U_{f}^{2} / H_{o}$. This determines a priori the order of magnitude of the dissipation $D_{\Omega}$ in the system. All contributions to the dissipation much smaller than this quantity will therefore be disregarded.

\section{DERIVATION OF $D_{\Omega}$ IN THE BUBBLE CASE}

In the case of a bubble in steady motion at velocity $U_{d} \mathbf{e}_{x}$ in a simple liquid, the boundary conditions at the interface of normal $\mathbf{n}$ are :

$$
\left\{\begin{array}{l}
u_{n}=U_{d} \mathbf{n} \cdot \mathbf{e}_{x} \\
\frac{\partial \mathbf{u}_{t}}{\partial n}=0 \\
\Delta P=\gamma \mathcal{C}
\end{array}\right.
$$

with $\mathbf{u}_{n}$ and $\mathbf{u}_{t}$ the projections of the velocity along the normal and in the tangential plane respectively, and $\mathcal{C}=\nabla \cdot \mathbf{n}$ is the curvature of the interface. The first condition is the mass conservation and the second expresses the continuity of the tangential stress, called the stress-free condition in the following. The third condition is the continuity of the normal 
stress at the interface, which boils down to the Laplace law in the low capillary number limit $[9,29,30]$.

These conditions, used as boundary conditions for the Stokes law fully determine the interface geometry and the velocity field $\mathbf{u}$. The dissipation in the domain $\Omega$ is purely viscous and the dissipation rate per unit volume is simply $\eta_{o}\left(\nabla u^{T}+\nabla u\right)^{2} / 2$.

Since it is not possible to solve the problem analytically over the whole domain $\Omega$, the latter is decomposed into different subdomains in which approximations can be performed.

In the domain limited by $R-H_{o}$ and $R_{\Omega}=R+H_{o}$ (see Fig. 4), the meniscus shape remains close to its equilibrium shape and is called the static meniscus. Its curvature

is $\mathcal{C}_{\text {stat }}=\left(\frac{1}{H_{o}}+\frac{\pi}{4} \frac{1}{R}\right)$ [31]. In this domain, the velocity gradients scale as $U_{d} / H_{o}$, the dissipation per unit volume as $\eta_{o} U_{d}^{2} / H_{o}^{2}$ and the global contribution to $D_{\Omega}$ is thus of the order of $\eta_{o} R U_{d}^{2} \ll \mathcal{P}$. The dissipation contribution from the static meniscus is therefore neglected in the following.

Closer to the wall, in contrast, typical length scales become much smaller and velocity gradients become non negligible. The film thickness in the region squeezed between the bubble and the wall is denoted $h(r, \theta)$. Its shape controls the dissipation and must therefore be computed to determine $D_{\Omega}$. Its curvature evolves from almost 0 in the central part of the bubble to its equilibrium value $\mathcal{C}_{\text {stat }}$ in the static meniscus, see Fig. 4. As long as the slope of the film thickness is small $(\|\nabla h\| \ll 1)$, $i$ e. close to the wall, lubrication approximations can be used to solve the dynamics. This region is called the lubrication film, in which most of the dissipation, neglected in the Taylor \& Saffman model, occurs.

\section{A. Viscous dissipation in the lubrication film}

The flow in the lubrication film is derived in the lubrication approximation, $\nabla^{2 D} p=$ $\eta_{o} \partial^{2} \mathbf{u} / \partial z^{2}$, with the velocity $\mathbf{u}=u_{r} \mathbf{e}_{r}+u_{\theta} \mathbf{e}_{\theta}$. Using the boundary conditions $\mathbf{u}(z=0)=\mathbf{0}$ and $\partial \mathbf{u} / \partial z(z=h)=\mathbf{0}$ (i.e. stress-free) we get:

$$
\begin{gathered}
\frac{1}{\eta_{o}} \frac{\partial p}{\partial r}=\frac{\partial^{2} u_{r}}{\partial z^{2}}=A \Longrightarrow u_{r}=\frac{A}{2}\left(z^{2}-2 h z\right) \\
\frac{1}{\eta_{o} r} \frac{\partial p}{\partial \theta}=\frac{\partial^{2} u_{\theta}}{\partial z^{2}}=A^{\prime} \Longrightarrow u_{\theta}=\frac{A^{\prime}}{2}\left(z^{2}-2 h z\right)
\end{gathered}
$$

In this study, we consider a pancake bubble with a radius much bigger than the half thickness of the Hele-Shaw cell, $R \gg H_{o}$. This geometrical constraint allows us to neglect 
the orthoradial pressure gradient and therefore the orthoradial velocity in the expression of the viscous dissipation. Indeed,the pressure gradient is given by the Laplace law in the lubrication approximation: $A=\frac{1}{\eta_{o}} \frac{\partial p}{\partial r}=-\frac{\gamma}{\eta_{o}} \frac{\partial^{3} h}{\partial r^{3}}$ and $A^{\prime}=\frac{1}{\eta_{o} r} \frac{\partial p}{\partial \theta}=-\frac{\gamma}{\eta_{o} r} \frac{\partial^{3} h}{\partial \theta \partial r^{2}}$ which verifies $A^{\prime} \ll A$. In the lubrication approximation, and for $R \gg H_{o}$, the dissipation in the lubrication film is thus approximated by

$$
D_{\mathrm{LF}} \simeq \frac{\eta_{o}}{2} \int_{\mathcal{V}}\left(\frac{\partial u_{r}}{\partial z}\right)^{2} \mathrm{~d} V
$$

where $\mathcal{V}$ is the volume of the lubrication film. As the radial pressure gradient in eq. (11) is given by the Laplace law in the lubrication approximation, $A=\frac{1}{\eta_{o}} \frac{\partial p}{\partial r}=-\frac{\gamma}{\eta_{o}} \frac{\partial^{3} h}{\partial r^{3}}$, the dissipation can be expressed as a function of the film profile $h(r, \theta)$. A $z$ integration on the top and bottom films gives

$$
D_{\mathrm{LF}}=\frac{2 \gamma^{2} R}{3 \eta_{o}} \int\left(\frac{\partial^{3} h}{\partial r^{3}}\right)^{2} h^{3} \mathrm{~d} \theta \mathrm{d} r
$$

in which the angular and the radial integrations are respectively performed within the intervals $[0,2 \pi]$ and $[0,+\infty[$, the infinite boundary for the integral being justified by the

asymptotic matching theory, $[9,30]$. Indeed, approaching the static meniscus, $\left(\frac{\partial^{3} h}{\partial r^{3}}\right)^{2} \rightarrow 0$, ensuring the convergence of the integral although the domain of integration is not bounded.

Equation (14) clearly shows the importance to determine the lubrication film thickness topography, which is intrinsically linked to the boundary condition at the interface, to calculate the dissipation. Such a topography, for a stress-free pancake bubble, has already been described theoretically by Burgess \& Foster [32] and numerically in [13]. In order to allow an easy reading of the present work, the results obtained in [13] to describe the topography of the film are recalled in the next section. The calculation of the resulting dissipation is presented in a section that follows.

\section{B. Lubrication film topography for a pancake bubble}

Burgess \& Foster derived, in the lubrication approximation, the following equation of the film profile for a pancake stress-free bubble [32]:

$$
\frac{\partial}{\partial r}\left[\frac{\partial^{3} h}{\partial r^{3}} h^{3}\right]=\frac{3 U_{d} \eta_{o}}{\gamma}\left(\cos \theta \frac{\partial h}{\partial r}-\sin \theta \frac{1}{r} \frac{\partial h}{\partial \theta}\right)
$$


This equation is an extension of the classical axisymmetric Bretherton's equation for the pancake geometry. This equation is valid in the limit $1 \gg \epsilon \gg C a[32]$.

The resolution of eq. (15) is performed by considering different regions of the lubrication film within which eq. (15) presents an asymptotic form. The corresponding simplified form of eq. (15) is then solved in each region.

Central domain. We first consider the central domain within which the curvature gradients are small. The left hand side member of eq. (15) can be neglected and the simplified form of eq. (15) is

$$
0=\frac{\partial h}{\partial x},
$$

in such a way that the film thickness deposited on the wall by the front meniscus remains unchanged until it reaches the rear meniscus. Consequently, the dissipation in this domain is negligible, as deduced from eq. (14).

The dynamical meniscus in contrast is characterized by large curvature variations. This region can be divided in three subregions: the front, the rear and the lateral dynamical meniscus. The front and rear meniscus regions are well separated as the dynamical meniscus extension, scaling as $H_{o} C a^{1 / 3} \ll H_{0}$. In the following, eq. (15) is solved in each of these subregions.

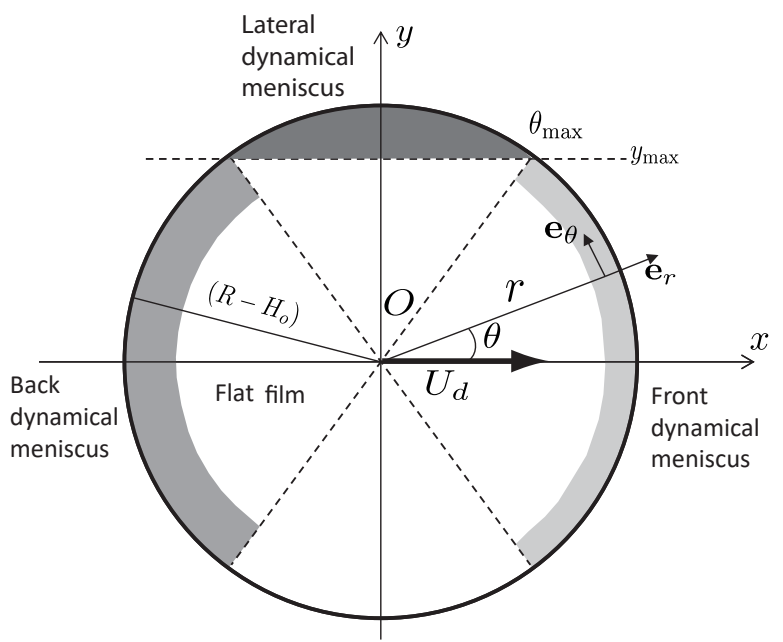

FIG. 5. Scheme of the droplet/bubble, of equatorial radius $R \gg H_{o}$ (pancake shape), moving at the velocity $U_{d} \mathbf{e}_{x}$ in the laboratory frame of reference.

Front/rear dynamical meniscus. Within the dynamical meniscus, since $H_{o} \ll R$, the condition $\cos \theta \partial_{r} h \ll \sin \theta \partial_{\theta} h / r$ is verified for $\theta \in\left[-\theta_{\max }, \theta_{\max }\right]$ (front dynamical meniscus) 
and $\theta \in\left[\pi-\theta_{\max }, \pi+\theta_{\max }\right]$ (rear dynamical meniscus), where $\theta_{\max } \approx 1.2$ [13], see Fig. 5 .

In these regions, eq. (15) is simplified into

$$
\frac{\partial}{\partial r}\left[\frac{\partial^{3} h}{\partial r^{3}} h^{3}\right]=\frac{3 U_{d} \cos \theta \eta_{o}}{\gamma} \frac{\partial h}{\partial r},
$$

corresponding to the classical Bretherton equation weighted by $\cos \theta$. Using the dimensionless variables,

$$
h=h_{\infty} H \text { and } \mathrm{r}=\mathrm{R}-\mathrm{H}_{\mathrm{o}}+\mathrm{h}_{\infty}(3 \mathrm{Ca}|\cos \theta|)^{-1 / 3} \xi
$$

leads to

$$
H^{3} \frac{\mathrm{d}^{3} H}{\mathrm{~d} \xi^{3}}= \pm(H-1)
$$

after one integration, with \pm corresponding respectively to the front and the rear meniscus.

This equation is solved in the front region by imposing the curvature $1 / H_{o}$ at large $\xi$, and a flat film at small $\xi$. Indeed, as $\epsilon \ll 1$, the curvature in the plan $(O x y)$ can be neglected in the static meniscus curvature $\mathcal{C}_{\text {stat }}=\frac{1}{H_{o}}\left(1+\frac{\pi}{4} \epsilon\right)$.

The front dynamical meniscus is the region which determines the thickness deposited in the central part of the lubrication film

$$
h_{\infty}^{\mathrm{BF}}(y)=1.34 H_{o} C a^{2 / 3}\left(1-\left(\frac{y}{R-H_{o}}\right)^{2}\right)^{1 / 3}
$$

corresponding to a weighted Bretherton solution. As shown by eq. (16), this thickness does not vary with $x$ in the central film region, and can thus be used as a boundary condition for the rear meniscus. The equation (17) is then solved at the rear by imposing the curvature $1 / H_{o}$ at large $\xi$, and a flat film of thickness $h_{\infty}^{\mathrm{BF}}(y)$ at small $\xi[9,32]$. The obtained dimensional and dimensionless thickness profiles are denoted $h^{f}$ and $H^{f}$ at the front and $h^{r}$ and $H^{r}$ at the rear. In principle, the use of the lubrication approximation to derive eq. (15) implies that the characteristic length over which $h$ varies, $H_{o} \mathrm{Ca}^{1 / 3}$, is small compared to $h \sim H_{o} C a^{2 / 3}$, leading to the condition $C a \ll 10^{-3}$ of validity of this approximation. In practice, comparison of the solution (20) for $y=0$ with experiments [33] and numerical simulation [34] of the film developing under an axisymmetric bubble translating in a capillary proved that this solution $1.34 H_{o} \mathrm{Ca}^{2 / 3}$ recovers the data on film thickness within a precision of $10 \%$ up to $C a=5 \times 10^{-3}$. 
Lateral dynamical meniscus. The lateral domains are defined by $|y|>y_{\max }$, with $y_{\max }=\left(R-H_{o}\right) \sin \theta_{\max }$, see Fig. 5. In this region, the orthoradial component of the viscous drag has to be considered and all three terms of eq. (15) must be taken into account. To build a master equation, ensuring that the terms in eq. (15) are of the same order of magnitude, Burgess \& Foster introduced the following rescalings [32]:

$$
r=R+H_{o} \delta_{r} r^{\prime} \quad z=H_{o} \delta_{z} z^{\prime} \quad h=H_{o} \delta_{z} h^{\prime} \quad \theta-\frac{\pi}{2}=\phi=\delta_{\theta} \phi^{\prime}
$$

where $\delta_{r}=\left(\epsilon^{1 / 2} C a\right)^{2 / 5}, \delta_{z}=\left(\epsilon^{1 / 2} C a\right)^{4 / 5}$ and $\delta_{\theta}=\epsilon^{1 / 2}\left(\epsilon^{1 / 2} C a\right)^{1 / 5}$. These quantities involve $\epsilon=H_{0} / R$ and the capillary number $C a$, under the assumption $C a \ll \epsilon \ll 1$.

Using these non dimensional variables and a first order expansion of the sin and cos terms around $\pi / 2$, eq. (15) becomes:

$$
\left(\frac{1}{3} h_{r^{\prime} r^{\prime} r^{\prime}}^{\prime} h^{\prime 3}+\phi^{\prime} h^{\prime}\right)_{r^{\prime}}=-h_{\phi^{\prime}}^{\prime}
$$

This equation in the lateral dynamical meniscus has been solved numerically, giving the dimensional solution noted $h^{\text {num }}$. The equation is solved for $-\phi_{\max }^{\prime} \leq \phi^{\prime} \leq \phi_{\max }^{\prime}$ with $\phi_{\max }^{\prime}=\left(\frac{\pi}{2}-\theta_{\max }\right) / \delta_{\theta}$ and $r_{\min }^{\prime} \leq r^{\prime} \leq r_{\max }^{\prime}$ with $r_{\min }^{\prime}=-18$ and $r_{\max }^{\prime}=26$. The profile $h^{\prime}\left(r^{\prime},-\phi_{\max }^{\prime}\right)$, corresponding to the solution $h^{f}\left(\theta_{\max }\right)$ for the front meniscus, is chosen as an initial condition and the profiles $h^{\prime}\left(r^{\prime}, \phi^{\prime}\right)$ for larger angles are obtained using a CrankNicholson scheme. The boundary conditions are a constant curvature of the interface in the meniscus, $\partial^{2} h / \partial r^{2}\left(r_{\max }\right)=1 / H_{o}$, and a connection to a flat film of constant thickness, $h\left(r_{\min }, \theta\right)=h\left(r_{\min }, \theta_{\max }\right)$ in [13].

Connecting together the solutions for the film profile in each region, leads to a catamaranlike shape of the lubrication film topography, see Fig. 6.

Now that all the results obtained for the topography of the lubrication film have been recalled, we will now determine the power dissipated in each of the different areas.

\section{Dissipation in the dynamical meniscus}

The main contribution to the total dissipation in $\Omega$ stems from the dynamical meniscus $[9$, 35]. Following the dynamical meniscus split performed in the previous section, we determine the dissipative contribution stemming from the front/rear and the lateral domains. 


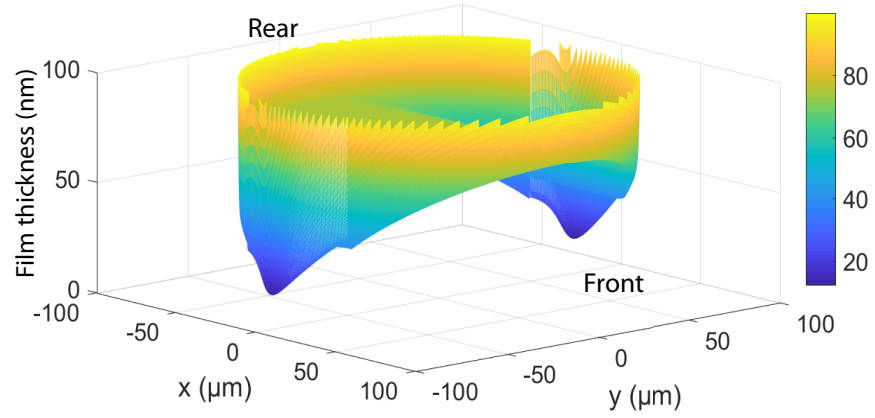

FIG. 6. Numerical topography of the lubrication film obtained by joining the profiles calculated in the different dynamical menisci with the central domain for $C a=7.2 \times 10^{-4}$.

Front/rear dynamical meniscus dissipation. According to eq. (14), the viscous dissipation rate in the front/rear dynamical meniscus is (summing the top and bottom contributions):

$$
D_{\text {men }}^{f, r}=\frac{2 \gamma^{2} R}{3 \eta_{o}} \int\left(\frac{\partial^{3} h^{f, r}}{\partial r^{3}}\right)^{2}\left(h^{f, r}\right)^{3} \mathrm{~d} \theta \mathrm{d} r
$$

where $h^{f}$ and $h^{r}$ are the solutions of eq. (17) in the front and the rear meniscus respectively. The angular integration interval in eq. (22) are $\left[-\theta_{\max }, \theta_{\max }\right]$ for the front domain and $\left[\pi-\theta_{\max }, \pi+\theta_{\max }\right]$ for the rear domain.

Using the dimensionless variables introduced in (18), the dissipation can be expressed in the front and the rear meniscus as a function of the master solution $H^{f / r}$ as:

$$
D_{\text {men }}^{f, r}=3^{2 / 3} \times 2 \frac{R \gamma^{2} C a^{5 / 3}}{\eta_{o}} \int_{\xi} \int_{\theta}\left(\frac{\partial^{3} H^{f, r}}{\partial \xi^{3}}\right)^{2} H^{3}|\cos \theta|^{5 / 3} \mathrm{~d} \xi \mathrm{d} \theta
$$

The integration of eq. (23) is performed using the solution $H^{r}$ and $H^{f}$ of eq. (19). It yields for the front and rear meniscus

$$
\begin{aligned}
& D_{\text {men }}^{f}=3.2 \times 3^{2 / 3} \times 1.2 \times \frac{R \gamma^{2} C a^{5 / 3}}{\eta_{o}} \\
& D_{\text {men }}^{r}=3.2 \times 3^{2 / 3} \times 1.16 \times \frac{R \gamma^{2} C a^{5 / 3}}{\eta_{o}}
\end{aligned}
$$

where $2 \int_{-\theta_{\max }}^{\theta_{\max }}|\cos \theta|^{5 / 3} \mathrm{~d} \theta=3.2$ and $I^{f}=\int_{\xi_{\min }}^{\xi_{\max }}\left(H^{f}-1\right)^{2}\left(H^{f}\right)^{-3} \mathrm{~d} \xi=1.2$ by integrating from $\xi_{\min }=-10$ to $\xi_{\max }=100$ guaranteeing to reach an asymptotic value of the integral. Similarly, we find $I^{r}=1.16$. The corresponding dissipations are plotted on Fig. 7 as a function of the capillary number and are found to be of almost equal contribution. 


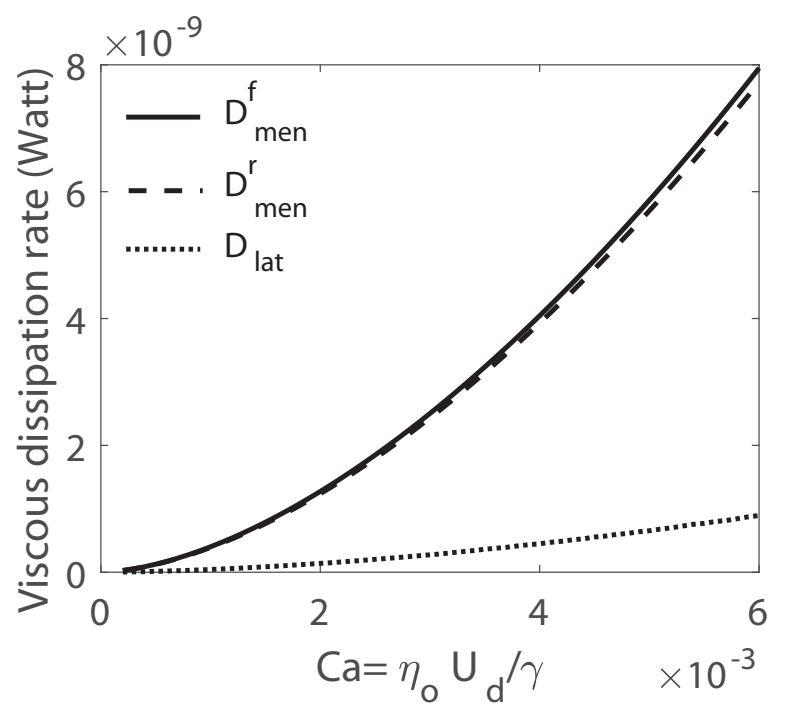

FIG. 7. Relative importance of all dissipative terms in System 1 as a function of the capillary number. $D_{\text {men }}^{f}, D_{\text {men }}^{r}$ and $D_{\text {lat }}$ are respectively the dissipations in the front (eq. (24)), the rear (eq. (25)) and the lateral (eq. (26)) dynamical meniscus.

Lateral dynamical meniscus dissipation. The dissipation in the lateral region (summing the two sides, and the top and bottom lubrication films) writes:

$$
D_{\text {lat }}=\frac{4 \gamma^{2} R}{3 \eta_{o}} \int\left(\frac{\partial^{3} h^{\text {num }}}{\partial r^{3}}\right)^{2}\left(h^{\text {num }}\right)^{3} \mathrm{~d} \theta \mathrm{d} r,
$$

where $h^{\text {num }}$ is the solution obtained numerically from eq. (21). The angular and radial integration intervals are respectively $\left[\theta_{\max }, \pi-\theta_{\max }\right]$ and $\left[r_{\min }, r_{\max }\right]$, with $r_{\min , \max }=R+$ $H_{o} \delta_{r} r_{\min , \max }^{\prime}$.

This dissipation is plotted as a function of the capillary number on Fig. 7. It represents 1/16 of the total dissipation, and can be considered negligible.

\section{Prediction of the bubble velocity}

The total dissipation $D_{\Omega}$ is obtained by summing the different contributions given by eqs. (24), (25), (26): $D_{\Omega}=D_{\text {men }}^{f}+D_{\text {men }}^{r}+D_{\text {lat }}$. The mobility is finally calculated using eq. (9) and is plotted in Fig. 8 with dashed lines to be compared to the experimental data. Remarkably, the obtained result is in very good agreement with the experimental data. In order to check that the dissipation in the lateral meniscus can be neglected, the prediction 
of the mobility without this contribution is plotted in solid lines in Fig. 8. Consistently with the result obtained in [11], the sole dissipation in the rear and front meniscus is sufficient to predict the bubble velocity.

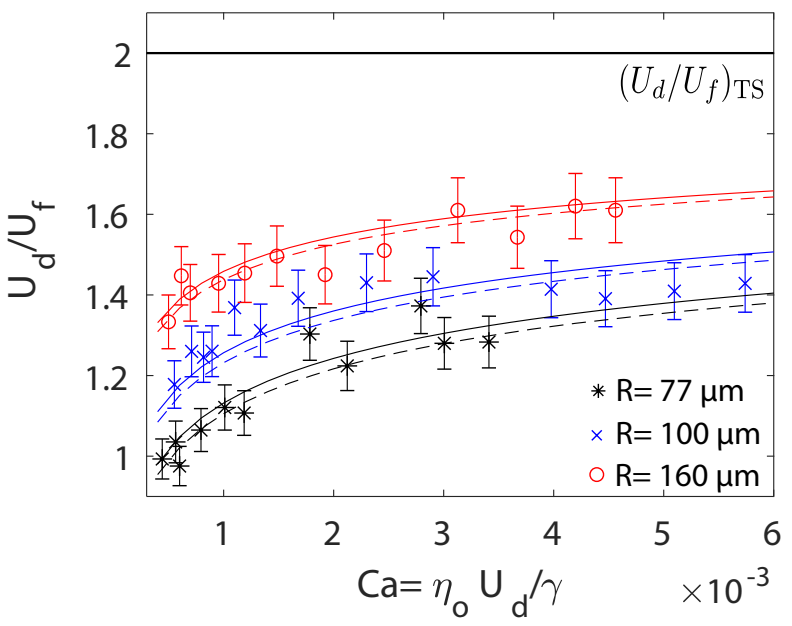

FIG. 8. Mobility $U_{d} / U_{f}$ as a function of $C a$ for bubbles (System 1) of different radii with $R=160$ $\mu \mathrm{m}(\circ), R=100 \mu \mathrm{m}(\times)$ and $R=77 \mu \mathrm{m}(\star)$. The colored lines are our theoretical predictions: the dashed lines include every source of dissipation (front, rear and lateral meniscus: $D_{\text {men }}^{f, r}$ and $D_{\text {lat }}$ ) whereas the solid lines do not take into account the contribution from the lateral meniscus shown to be negligible. The horizontal line is Taylor \& Saffman prediction $\left(U_{d} / U_{f}\right)_{\mathrm{TS}}=2[6]$.

The model provides a natural interpretation of the mobility dependency with the capillary number and bubble radius. Indeed, eq. (9) shows that the dissipation close to the bubble must be compared to the reference dissipation $D_{\text {ref }}=\eta_{o} U_{d}^{2} R^{2} / H_{o}$. The correction to the Taylor \& Saffman prediction becomes negligible for $D_{\Omega} \ll D_{\text {ref }}$.

All correction terms scale as the perimeter $R$ of the bubble, whereas the reference dissipation scales as the bubble area $R^{2}$, the relative importance of these terms thus decreases at bigger size. Consistently, the observed discrepancy with TS model decreases at bigger bubble diameter. Similarly, meniscus friction on a solid wall is generically sublinear with the velocity [9], and the correction terms thus have a dependency in $U_{d}$ smaller than 2 . The reference dissipation in contrast varies linearly with $U_{d}^{2}$. This explains why the discrepancy also decreases as the velocity increases.

As a whole, this section validated our approach in the case of a bubble for a stress-free boundary condition at the interface. In the following section, we are interested in the case 
of a drop in an aqueous solution containing surfactant.

\section{DERIVATION OF $D_{\Omega}$ FOR SURFACTANT LADEN DROPLET OF LOW VIS- COSITY.}

In this case, an additional term arises from the dissipation in the drop bulk, due to its non vanishing viscosity. Otherwise, most of the dissipative terms are unchanged. Indeed, for the specific system discussed here, it was shown experimentally in [13] that the boundary condition at the interface is stress-free in the front and central regions. This indicates that (i) the viscous stress due to the inner phase remains small at the oil/water interface, in agreement with Park \& Homsy prediction, stating that the interface of a viscous droplet remains stress-free as long as the viscosity ratio between the inner and outer phase verifies $\eta_{i} / \eta_{o} \ll C a^{-1 / 3}[30]$; (ii) the surfactants have no mechanical effects in these regions. This is not true anymore close to the rear meniscus where the surfactant molecules accumulate inducing Marangoni stresses and modifying the boundary conditions at the interface. The dissipation in this domain thus requires a specific discussion.

\section{A. Inner droplet and central part of the lubrication film}

The derivation of the flow profile in the inner droplet and in the central part of the lubrication film implies to know the kinematic boundary condition $\mathbf{U}_{i}$ at the interfaces located at $z=h_{\infty}$ and $z=2 H_{o}-h_{\infty}$. In the central region, a stress-free boundary condition was deduced in [13], insuring the absence of Marangoni stress and the continuity of the tangential viscous stresses at the interfaces. Consequently, a scaling law for $U_{i}$ can be established by balancing the inner and outer viscous stress $\eta_{i}\left(U_{d}-U_{i}\right) / H_{o} \sim \eta_{o} U_{i} / h_{\infty}$,

which yields $U_{i} \sim \frac{\alpha}{1+\alpha} U_{d}$, with $\alpha=\frac{\eta_{i}}{\eta_{o}} \frac{h_{\infty}}{H_{o}}$. As $\eta_{i} \leq \eta_{o}$ and $h_{\infty} \ll H_{o}, \alpha \ll 1$ and $U_{i} \sim \alpha U_{d} \ll U_{d}$; the interface is stress-free.

Within the droplet, the flow can be well described by a Poiseuille flow of uniform depthaveraged velocity $U_{d} \mathbf{e}_{x}[4]$. Considering $h_{\infty} \ll H_{o}$ and $U_{i} \ll U_{d}$, yields:

$$
D_{\text {drop }}=\frac{6 \eta_{i} U_{d}^{2} \pi R^{2}}{H_{o}}
$$

This term is plotted in Fig. 9 . 


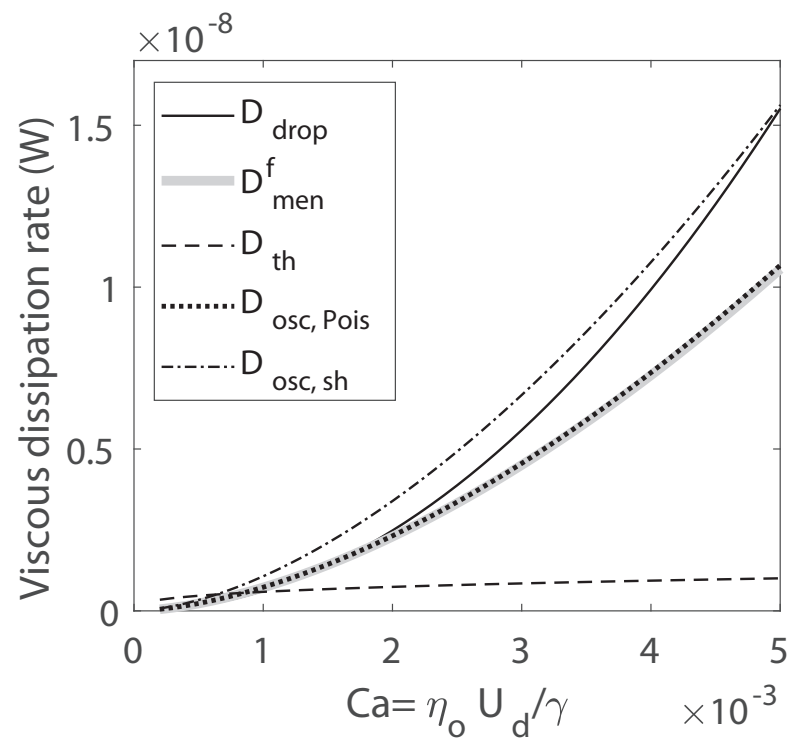

FIG. 9. Relative importance of all dissipative terms in System 2 as a function of the capillary number. $D_{\text {drop }}, D_{\text {men }}^{f}$ and $D_{\text {th }}$ are respectively the dissipations in the drop (eq. (27)), in the front dynamical meniscus (eq. (24)) and in the thickening region (eq. (32)). $D_{\text {osc,Pois }}$ and $D_{\text {osc,sh }}$ are the Poiseuille (eq. (37)) and shear (eq. (38)) contributions to the dissipation in the oscillating region.

Using the same assumption, the dissipation in the flat film of volume $R^{2} h_{\infty}$ scales as:

$$
D_{\text {film }}=\eta_{o}\left(\frac{\eta_{i}}{\eta_{o}}\right)^{2}\left(\frac{R^{2} U_{d}^{2}}{H_{o}}\right)\left(\frac{h_{\infty}}{H_{o}}\right) .
$$

This contribution is $h_{\infty} / H_{o}$ times smaller than the reference dissipation $D_{\text {ref }}$ (see eq. (9)) and is therefore neglected in the following.

\section{B. Front meniscus}

In the front dynamical meniscus, the thickness profile is found to be in good agreement with the solution $h^{f}$, evidencing a stress-free boundary condition interface in this domain [13]. As a consequence, the dissipation in the corresponding domain is given by eq. (24).

\section{Rear meniscus profile and interfacial boundary condition}

The thickness profile at the rear meniscus could not be reproduced by the stress-free solution $h^{r}$ of eq. (17), meaning that an additional stress appears at the interface. The model 
proposed in [13] to predict the film profile and the interfacial boundary condition at the rear requires dividing the rear dynamical meniscus into two regions (see Fig 10.a): a thickening region located at the transition between the flat film and the rear bump, and an oscillating region. In the first region, the Laplace pressure is neglected and the surfactant transport is solved assuming that the flow is solely along the $x$-direction. We found experimentally that the Marangoni stress is established on a characteristic distance $l \simeq 8 \mu \mathrm{m}$ in [13]. Given that this stress is established over this characteristic length in the $x$-direction and $R$ in the $y$ direction, verifying $l \ll R$, the film profile is mostly determined by the important Marangoni stress developing in the $x$-direction. In the second region, a full stiffening of the interface is assumed leading to a uniform interfacial velocity as in the stagnant cap situation [36]. The lubrication equations are solved, including the Laplace pressure contribution. Before establishing any dissipation calculation, we recall the interfacial and flow properties in these two regions.
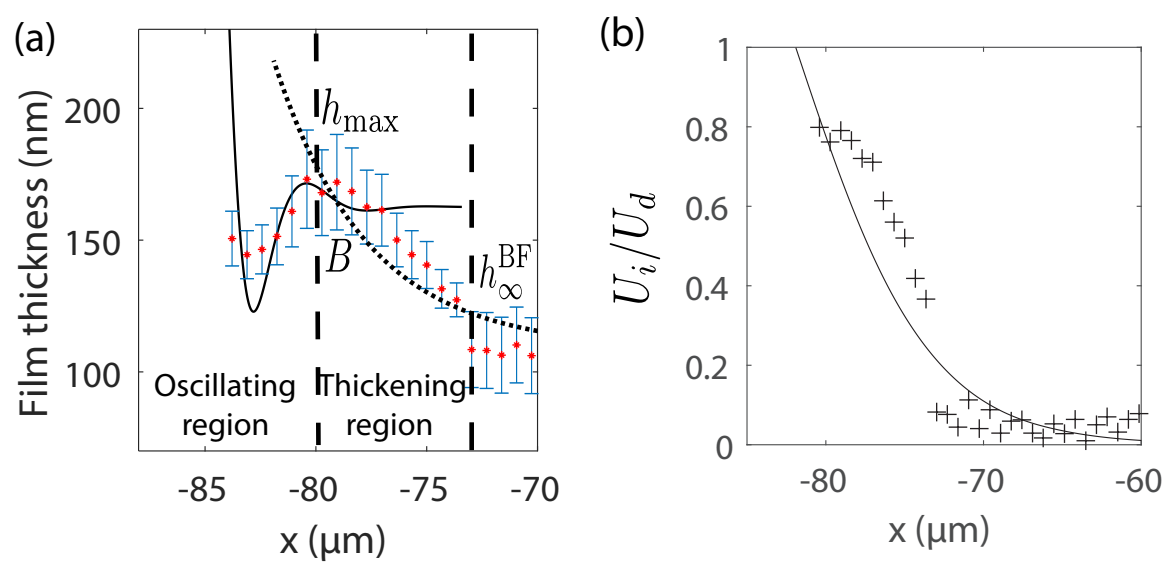

FIG. 10. (a) Oscillation at the rear meniscus. Longitudinal $(y=0)$ thickness profile for $C a=$ $1.3 \times 10^{-3}$. The film thickness at large $x$ is $h_{\infty}^{\mathrm{BF}}$ and the local maximum is $h_{\max }$. The dotted line shows the film profile deduced using the surfactant mass transport model with $M a=1.6$. The solid line shows the theoretical prediction from eq. (34) using $U_{i}(B)=2 U_{d}\left(1-h_{\infty}^{\mathrm{BF}} / h_{\max }\right)$; (b) Interfacial velocity in the thickening region normalized by the droplet velocity for $C a=1.3 \times 10^{-3}$. The solid line is the interfacial velocity obtained from the surfactant mass transport model with $M a=1.6$. [Extracted from [13].]

Thickening region. The Laplace pressure is neglected in the thickening region, the flow is thus a simple shear flow and the mass balance provides a relationship between the experi- 
mental film thickness and the interfacial velocity $U_{i}(x) \mathbf{e}_{x}=2 U_{d}\left[1-h_{\infty}^{\mathrm{BF}}(y) / h(x, y)\right] \mathbf{e}_{x}$. This interfacial velocity is plotted on Fig. 10.b. At the peak of the experimental bump located at the transition with the oscillating region we measured $U_{i}\left(x_{B}\right)=2 U_{d}\left(1-h_{\infty}^{\mathrm{BF}} / h_{\max }\right) \approx 0.8 U_{d}$, regardless of the capillary number.

Coupling this shear flow to the surfactant mass transport equation, for which the driving transport mechanisms are interfacial and bulk convections, the interfacial velocity and the thickness profile in the thickening region write:

$$
\begin{gathered}
U_{i}^{\mathrm{th}}(x)=U_{d}\left[1-\tanh \left(\frac{\left(x-x_{0}\right) C a}{2 M a h_{\Gamma}}\right)\right] \\
h^{\mathrm{th}}(x, y)=2 h_{\infty}^{\mathrm{BF}}(y) /\left[1+\tanh \left(\frac{\left(x-x_{0}\right) C a}{2 M a h_{\Gamma}}\right)\right]
\end{gathered}
$$

where $M a=\left|\partial_{\Gamma} \gamma\right| \Gamma_{\infty} / \gamma$ is the Marangoni number with $\Gamma$ the surfactant surface concentration, $\Gamma_{\infty}$ the surfactant surface concentration at equilibrium in the flat film and $h_{\Gamma}=\Gamma_{\infty} / C_{\infty}=3 \mathrm{~nm}$ is the surfactant depletion length, with $C_{\infty}$ the bulk concentration in the flat film. The reference position $x_{0}$ is fitted on the experimental data so that $h^{\exp }\left(x_{B}, 0\right)=h^{\text {th }}\left(x_{B}, 0\right)$. These expressions quantitatively reproduce the experimental film thickness and the interfacial velocity for a single value of the model's parameter $M a=1.6 \pm 0.2$, see Fig. 10.

In the lubrication approximation, the dissipation in the thickening region is given by:

$$
D_{\mathrm{th}}=2 \eta_{o} \int_{x_{B}}^{+\infty} \int_{-y_{\max }}^{y_{\max }} \int_{0}^{h(x, y)}\left(\frac{\partial u_{x}}{\partial z}\right)^{2} \mathrm{~d} x \mathrm{~d} y \mathrm{~d} z
$$

where $x_{B}$ is the coordinate of the bump chosen arbitrarily as the frontier between the thickening and oscillating region. As $\frac{U_{i}\left(x_{B}\right)}{U_{d}}=0.8$, we have $x_{B}=\frac{2 M a h_{\Gamma}}{C a} \operatorname{Argth}(0.2)+x_{0}$. The numerical factor 2 in eq. (31) stands for the top and bottom thickening regions. The Couette flow in this region, along with eq. (29) allow to give a simple expression for $D_{\text {th }}$ :

$$
D_{\mathrm{th}}=\eta_{o} U_{i}^{2}\left(x_{B}\right) \frac{M a h_{\Gamma}}{C a} \int_{-y_{\max }}^{y_{\max }} \frac{\mathrm{d} y}{h_{\infty}^{\mathrm{BF}}(y)} .
$$

This contribution is plotted on Fig. 9 and is found to be negligible compared to the one calculated previously, namely $D_{\text {drop }}$.

Oscillating region. We now turn to the oscillating region, where both the Laplace pressure effect and the presence of surfactant at the interface contribute, the later one being 
modeled by the homogeneous surface velocity, $U_{i}\left(x_{B}\right)=2 U_{d}\left(1-h_{\infty}^{\mathrm{BF}} / h_{\max }\right) \mathbf{e}_{x}$. Under this assumption

$$
\frac{1}{\eta_{o}} \frac{\partial p}{\partial r}=\frac{\partial^{2} u_{r}}{\partial z^{2}}=A \Longrightarrow u_{r}=\frac{A}{2}\left(z^{2}-h_{\mathrm{inc}} z\right)+\frac{U_{i}\left(x_{B}\right) \cos \theta z}{h_{\mathrm{inc}}}
$$

The governing equation for the thickness profile $h_{\text {inc }}$ is thus, for $|y|<y_{\max }$ :

$$
\frac{\partial}{\partial r}\left[\frac{\partial^{3} h_{\text {inc }}}{\partial r^{3}} h_{\text {inc }}^{3}\right]=\frac{3 U_{d} \eta_{o}}{\gamma}\left(4-\frac{2 U_{i}\left(x_{B}\right)}{U_{d}}\right)\left(\cos \theta \frac{\partial h_{\text {inc }}}{\partial r}\right)
$$

In eq. (34) it is assumed that the surface tension variation, ensuring the uniform interfacial velocity, remains small in comparison with the equilibrium surface tension value. In that case, the Laplace pressure is determined from the non modified surface tension $\gamma$, which is the classical Bretherton approximation, see eq. (33) in [9].

The film profile $h_{\text {inc }}^{r}(r, \theta)$ is determined by imposing the curvature $1 / H_{o}$ at large $r$ and the film thickness at small $r, h_{\infty}^{*}$ is fitted so that the maximum experimental height $h_{\max }$ (at $x_{B}$ ) is recovered by the model, i.e. $\quad h_{\infty}^{*}=h_{\max } / 1.064$. This numerical prediction for $\theta=\pi$ is plotted on Fig. 10.a (solid line) and is in very good agreement with the experimental back oscillation, thus validating that the interface velocity does not vary significantly once it reaches values as big as $U_{i}\left(x_{B}\right)$. Similarly to the stress-free case, eq. (34) can be non dimensionalized at the rear and eq. (19) can be recovered using the following non dimensionless variables

$$
h_{\text {inc }}=h_{\infty}^{*} H \text { and } \mathrm{r}=\mathrm{R}-\mathrm{H}_{\mathrm{o}}+\mathrm{h}_{\infty}^{*}\left[6\left(2-\mathrm{U}_{\mathrm{i}}\left(\mathrm{x}_{\mathrm{B}}\right) / \mathrm{U}_{\mathrm{d}}\right) \mathrm{Ca}|\cos \theta|\right]^{-1 / 3} \xi
$$

$H^{r}$ is the dimensionless solution of eq. (19), for an incompressible interface at the rear of the droplet.

In the oscillating region, the viscous dissipation rate is, after integration on $z$ on the top and bottom meniscus:

$$
D_{\mathrm{osc}}=\frac{\gamma^{2} R}{6 \eta_{o}} \int\left(\frac{\partial^{3} h_{\mathrm{inc}}^{r}}{\partial r^{3}}\right)^{2}\left(h_{\mathrm{inc}}^{r}\right)^{3} \mathrm{~d} \theta \mathrm{d} r+2 \eta_{o} U_{i}\left(x_{B}\right)^{2} R \int \frac{1}{h_{\mathrm{inc}}^{r}} \mathrm{~d} \theta \mathrm{d} r=D_{\mathrm{osc}, \text { Pois }}+D_{\mathrm{osc}, \mathrm{sh}}
$$

The angular integration interval is $\left[\pi-\theta_{\max }, \pi+\theta_{\max }\right]$. This expression shows that the flow in this region results from the combination of a Poiseuille flow induced by the capillary suction $D_{\text {osc,Pois }}$ (first term) and a simple shear induced by the velocity difference between 
the interface, of uniform velocity $U_{i}\left(x_{B}\right)$, an the wall, $D_{\text {osc,sh }}$ (second term). The Poiseuille flow contribution can be expressed as a function of the master solution $H^{r}$ as

$$
D_{\text {osc,Pois }}=4^{2 / 3}\left[1-U_{i}\left(x_{B}\right) /\left(2 U_{d}\right)\right]^{5 / 3} 3.2 \times 3^{2 / 3} \frac{R \gamma^{2} C a^{5 / 3}}{\eta_{o}} I^{r}
$$

where $I^{r}=\int\left(H^{r}-1\right)^{2}\left(H^{r}\right)^{-3} \mathrm{~d} \xi=1.16$ is integrated between $\xi_{\min }=-10$ and $\xi_{\max }=100$.

This contribution is plotted on Fig. 9. For $U_{i}\left(x_{B}\right)=0.8 U_{d}, 4^{2 / 3}\left[1-U_{i}\left(x_{B}\right) /\left(2 U_{d}\right)\right]^{5 / 3}=1.08$ and the Poiseuille dissipation in the rear oscillating region is, by coincidence, extremely close to the dissipation at the front in the stress-free case $D_{\text {men }}^{f}$, see eq. (24).

The simple shear contribution for the oscillating region is given by

$$
D_{\text {osc,sh }}=\frac{5.31}{6^{1 / 3}} \frac{\eta_{o} U_{i}\left(x_{B}\right)^{2} R}{\left(2 C a-C a_{i}\right)^{1 / 3}} I_{2}
$$

with $I_{2}=\int_{\xi_{B}}^{\xi_{\max }} \frac{1}{H^{r}} \mathrm{~d} \xi$ and $C a_{i}=\eta_{o} U_{i}\left(x_{B}\right) / \gamma$. In contrast with the previous integral, this term varies with the boundary value $\xi_{B}$, which represents the frontier $x_{B}$ between the oscillating and thickening regions consistently with integral boundary of eq. (31). The contribution of this dissipation happens to be the most important in the system and shows the importance of taking into account the incompressible feature of the interface.

\section{Prediction of the drop velocity}

The theoretical mobilities $U_{d} / U_{f}$ are deduced from eq. (9) by keeping the relevant terms in $D_{\Omega}: D_{\text {men }}^{f}, D_{\text {drop }}, D_{\text {th }}$ and $D_{\text {osc }}$.

The mobilities obtained by considering the relevant terms are plotted as solid lines on Fig. 11. It is noticeable that without any fitting parameter the model reproduces quantitatively the experimental data on the major part of the $C a$ range. In order to validate the relevance of the refinements performed for System 2, the theoretical mobilities for an entire stress-free interface are also plotted on Fig. 11 (dashed lines). It is clear that a stress-free model leads to an overestimation of the mobility by $30 \%$. Consequently, a stiffening of the interface, even over a small portion of the interface at the rear (5\% of the film), can significantly influence the droplet velocity. This result clearly shows that the boundary condition needs to be determined at the whole interface if one wants to predict the droplet velocity. 


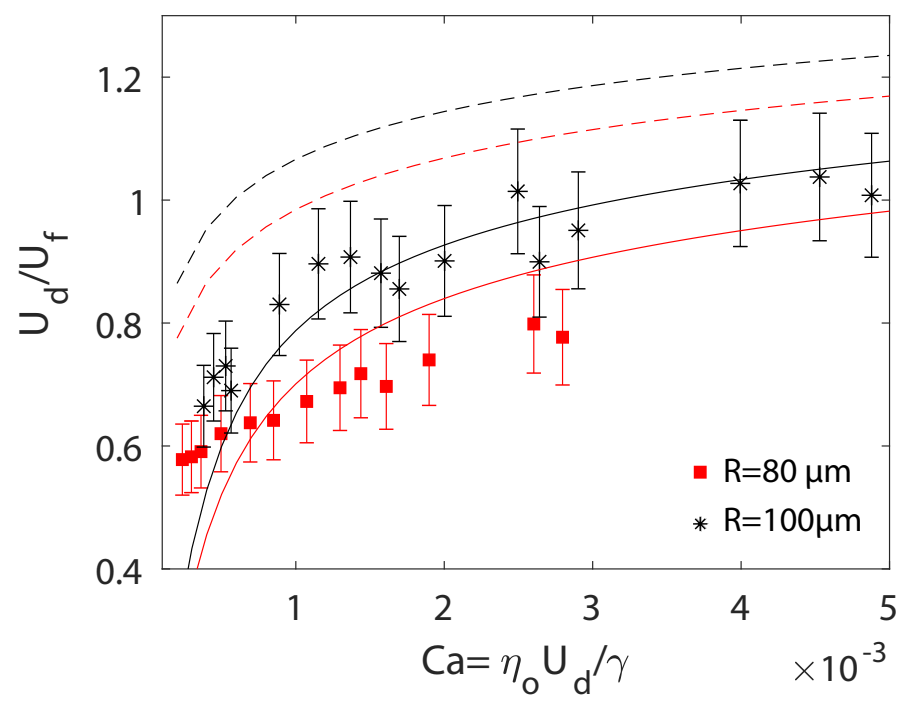

FIG. 11. Mobility $U_{d} / U_{f}$ of droplets (System 2) of different radii, with $R=100 \mu \mathrm{m}(\star)$ and $R=80$ $\mu \mathrm{m}(\mathbf{\square})$. The colored lines are our theoretical predictions without $D_{\text {lat }}$ : solid lines take into account the stiffening of the interface at the rear of the droplet. The dashed lines corresponds to the full stress-free model.

\section{CONCLUSION}

In this paper, we report experimental measurements of droplets velocities in microdevices. We rationalize quantitatively our results by considering non-homogeneous boundary conditions stemming from very small surface tension variations due to an accumulation of surfactant at the rear meniscus. This is especially critical in the interface domain close to the solid wall and this sensitivity is probably at the origin of the usual difficulties to reproduce drop and bubble velocities in confined situation with simple models. An important contribution of this work is to explain, for a given chemical system, all the dissipative processes together with their spatial localization. Especially, we show that the one influenced by the presence of surfactant is localized at the rear in the most compressive part of the interface. The good agreement obtained in the present paper using these data confirms the validity of the whole approach. Our work reveals that, unless the interface is clean, the full prediction of the drop or bubble velocity would require a deeper understanding of surfactant transport as a function of physico-chemical properties of the solutions. Finally, on the basis of the model that we developed, whether or not surfactants accumulate can be directly deduced from the droplet mobility. 


\section{Acknowledgments}

The authors wish to thank Lola Ciapa for technical support. This work was supported by CNRS, IPGG (Equipex ANR-10-EQPX-34 and Labex ANR-10-LABX-31), PSL (Idex ANR-10-IDEX-0001-02), ESPCI Paris, and Agence Nationale de la Recherche (ANR) under the grants ANR-13-BS09-0011 and ANR-18-CE09-0029.

[1] A. Huerre, O. Theodoly, A. Leshansky, M.-P. Valignat, I. Cantat, and M.-C. Jullien, "Droplets in microchannels: dynamical properties of the lubrication film," Physical review letters 115, $064501(2015)$.

[2] Y. Ling, J.-M. Fullana, S. Popinet, and C. Josserand, "Droplet migration in a hele-shaw cell: Effect of the lubrication film on the droplet dynamics," Physics of Fluids 28, 062001 (2016).

[3] C. Baroud, F. Gallaire, and R. Dangla, "Dynamics of microfluidic droplets," Lab on a Chip 10, 2032-2045 (2010).

[4] S. Lee, F. Gallaire, and C. Baroud, "Interface-induced recirculation within a stationary microfluidic drop," Soft Matter 8, 10750-10758 (2012).

[5] K. Okumura, "Viscous dynamics of drops and bubbles in hele-shaw cells: Drainage, drag friction, coalescence, and bursting," Advances in colloid and interface science (2017).

[6] G. Taylor and P. G. Saffman, "A note on the motion of bubbles in a hele-shaw cell and porous medium," The Quarterly Journal of Mechanics and Applied Mathematics 12, 265-279 (1959).

[7] A. R. Kopf-Sill and G. M. Homsy, "Bubble motion in a hele shaw cell," Physics of Fluids 31, 18-26 (1988).

[8] C.-W. Park, S. R. K. Maruvada, and D.-Y. Yoon, "The influence of surfactant on the bubble motion in hele-shaw cells," Physics of Fluids 6, 3267-3275 (1994).

[9] F. P. Bretherton, "The motion of long bubbles in tubes," Journal of Fluid Mechanics 10, 166-188 (1961).

[10] S. R. K. Maruvada and C.-W. Park, "Retarded motion of bubbles in hele-shaw cells," Physics of fluids 8, 3229-3233 (1996).

[11] E. Reyssat, "Drops and bubbles in wedges," Journal of Fluid Mechanics 748, 641-662 (2014).

[12] L. Zhu and F. Gallaire, "A pancake droplet translating in a hele-shaw cell: lubrication film and flow field," Journal of Fluid Mechanics 798, 955-969 (2016). 
[13] B. Reichert, A.l Huerre, O. Theodoly, M.-P. Valignat, I. Cantat, and M.-C. Jullien, "Topography of the lubrication film under a pancake droplet travelling in a hele-shaw cell," Journal of Fluid Mechanics 850, 708-732 (2018).

[14] G.J. Hirasaki and J. B. Lawson, "Mechanisms of foam flow in porous media : apparent viscosity in smooth capillaries," Society of Petroleum Engineers Journal 25, 176-188 (1985).

[15] J. Ratulowski and H.-C. Chang, "Marangoni effects of trace of impurities on the motion of long gas bubbles in capillaries," Journal of Fluid Mechanics 210, 303-328 (1990).

[16] K. J. Stebe and D. Barthès-Biesel, "Marangoni effects of adsorption/desorption controlled surfactants on the leading end of an infinitely long bubble in a capillary," Journal of Fluid Mechanics 286, 25-48 (1995).

[17] C. W. Park, "Influence of soluble surfactants on the motion of a finite bubble in a capillary tube," Physics of Fluids 4, 2335-2347 (1992).

[18] J.W.M. Bush, "The anomalous wake accompanying bubbles rising in a thin gap: a mechanically forced marangoni flow," Journal of Fluid Mechanics 352, 283-303 (1997).

[19] J. Chen and K.J. Stebe, "Surfactant-induced retardation of the thermocapillary migration of a droplet," Journal of Fluid Mechanics 340, 35-59 (1997).

[20] I. Cantat and B. Dollet, "Liquid films with high surface modulus moving in tubes: dynamic wetting film and jumpy motion," Soft Matter 8, 7790 (2012).

[21] V. Miralles, E. Rio, I. Cantat, and M.-C. Jullien, "Investigating the role of a poorly soluble surfactant in a thermally driven 2d microfoam," Soft matter 12, 7056-7062 (2016).

[22] Y. Xia and G.M. Whitesides, "Soft lithography," Annual review of materials science 28, 153184 (1998).

[23] N. Taccoen, On the long-term stability of foams: strength of an armored bubble and emergence of global dis Ph.D. thesis, Ecole Doctorale de l'Ecole Polytechnique (2015).

[24] T. Thorsen, R. W. Roberts, F. H. Arnold, and S. R. Quake, "Dynamic pattern formation in a vesicle-generating microfluidic device," Physical review letters 86, 4163 (2001).

[25] F. Gallaire, P. Meliga, P. Laure, and C. Baroud, "Marangoni induced force on a drop in a hele shaw cell," Physics of Fluids 26, 062105 (2014).

[26] R. Dangla, 2D droplet microfluidics driven by confinement gradients, Ph.D. thesis, Ecole Doctorale de l'Ecole Polytechnique (2012).

[27] E. Guyon, J.-P. Hulin, and L. Petit, Hydrodynamique physique (EDP science, Paris, 2001). 
[28] A. Nadim, A. Borhan, and H. Haj-Hariri, "Tangential stress and marangoni effects at a fluid-fluid interface in a hele-shaw cell," Journal of colloid and interface science 181, 159-164 (1996).

[29] S. R. Hodges, O. E. Jensen, and J. M. Rallison, "The motion of a viscous drop trhough a cylindrical tube," Journal of Fluid Mechanics 501, 279 (2004).

[30] C.-W. Park and G. M. Homsy, "Two-phase displacement in hele shaw cells: theory," Journal of Fluid Mechanics 139, 291-308 (1984).

[31] P. S. de Laplace, Traité de mécanique céleste: Théorie de l'action capillaire. Suppl (Courcier, 1806).

[32] D. Burgess and M. R. Foster, "Analysis of the boundary conditions for a hele-shaw bubble," Physics of Fluids A: Fluid Dynamics 2, 1105-1117 (1990).

[33] P. Aussillous and D. Quéré, "Quick deposition of a fluid on the wall of a tube," Physics of Fluids 12, 2367-2371 (2000).

[34] G. Balestra, L. Zhu, and F. Gallaire, "Viscous taylor droplets in axisymmetric and planar tubes: from brethertonâs theory to empirical models," Microfluidics and Nanofluidics 22, 67 (2018).

[35] I. Cantat, "Liquid meniscus friction on a wet wall: bubbles, lamellae and foams," Physics of Fluids 25, 031303 (2013).

[36] B. Cuenot, J. Magnaudet, and B. Spennato, "The effects of slightly soluble surfactants on the flow around a spherical bubble," Journal of Fluid Mechanics 339, 25-53 (1997). 